\title{
¿Los Estándares Internacionales de Información Financiera -NIIF- convergen con el enfoque del contexto actual de Colombia?
}

\author{
Natalia Andrea Rodríguez Rodríguez ${ }^{1}$ \\ Jonatan Fernando Neiva Ardila ${ }^{2}$ \\ Jhon César Junior Páez Duarte ${ }^{3}$
}

Recibido: 21 de julio 2015

Aprobado: 15 de noviembre 2015

\begin{abstract}
Rodríguez, N., Neiva, J. \& Páez, J. (2015). ¿Los Estándares Internacionales de Información Financiera -NIIF- convergen con el enfoque del contexto actual de Colombia? Activos 25, 187-202
\end{abstract}

Clasificación JEL: G38, G39.

\section{Resumen}

La Ley 1314 de 2009 estableció que Colombia realizaría un proceso de convergencia con estándares internacionales de aceptación mundial, aunque

1 Estudiante Noveno semestre, Universidad Nacional de Colombia Semillero: CENIIF-UN, Comité de Estudio en Normas Internacionales de Información Financiera Correo electrónico naarodriguezro@unal.edu.co

2 Estudiante Noveno semestre, Universidad Nacional de Colombia Semillero: CENIIF-UN, Comité de Estudio en Normas Internacionales de Información Financiera Correo electrónico jfneivaa@unal.edu.co

3 Estudiante Noveno semestre, Universidad Nacional de Colombia Semillero: CENIIF-UN, Comité de Estudio en Normas Internacionales de Información Financiera Correo electrónico: jjpaezd@unal.edu.co 
dicho proceso no se cumple realmente dado que el mercado económico colombiano y la evolución de la ciencia contable en Colombia no ha tenido el mismo nivel de países donde se aplican las NIIF desde hace algún tiempo. Por tanto, se hace necesario un cambio de pensamiento y tratamiento en temas contables, para ello este artículo muestra cómo la implementación de Estándares Internacionales de Información Financiera -NIIF- pueden garantizar el mejoramiento de la información, sin converger completamente al contexto colombiano.

\title{
Palabras clave
}

Convergencia, Normas Internacionales de Información Financiera (NIIF), neoliberalismo, modelo contable, valor razonable.

\section{Rodríguez, N., Neiva, J. \& Páez, J. (2015). Does the International Financial Reporting Standard -IFRS- coincide with the approach of the current context of Colombia? Activos 25, 187-202}

\begin{abstract}
Act 1314 of 2009 established that Colombia would carry out a convergence process with international standards accepted worldwide, however, this process has not been accomplished yet because the Colombian market and the evolution of accounting science in Colombia has not achieved the same level as countries where IFRS has been applied for some time. Therefore, there must be a change in the way of thinking and treatment of accounting issues, in this regard, this article shows how the implementation of International Financial Reporting Standard - IFRS - can ensure improved information, without fully converging the Colombian context.
\end{abstract}

\section{Keywords}

Convergence, International Financial Reporting Standard (IFRS), neoliberalism, accounting model, fair value. 
Rodríguez, N., Neiva, J. \& Páez, J. (2015). Est-ce que les normes internationales d'information financière - NIIF - convergent avec l'approche du contexte actuel de la Colombie? Activos 25, 187-202

\section{Résumé}

La loi 1314 du 2009 a établi que la Colombie procéderait à un processus de convergence avec les standards internationaux reconnues dans le monde, bien que ce processus ne soit pas vraiment établi à cause du marché économique colombien et à que lévolution des sciences de la comptabilité en Colombie nont pas le même niveau que dans les pays où les NIIF sont appliquées depuis un certain temps. Par conséquent, un changement de mentalité et de traitement des questions de comptabilité est nécessaire. Pour cette raison, cet article montre comment l'implémentation des normes international d'information financière - NIIF - peut assurer une meilleure information sans converger pleinement le contexte colombien.

\section{Mots clés}

Convergence, Cormes International d'information financière.

\section{Introducción}

Colombia históricamente ha sido un país subdesarrollado, y esto se debe a que desde sus inicios el país fue tratado como colonia española, por lo cual el actuar y las decisiones se tomaron con base a las imposiciones que traía consigo su categoría. Al lograr la independencia y dar inicio como Nación y República se observa que aún se continúa con la dependencia de modelos y tratamientos de un grupo de países que se denominan como desarrollados en diversos aspectos, es de esta manera como se tiene que Colombia por necesidad de continuar y lograr un desarrollo a nivel económico requiere adaptarse a requerimientos internacionales para atraer así inversión por parte de aquellos que son considerados desarrollados. Estos 
cambios presentan su máximo auge desde el siglo pasado, donde se tiene que a partir de los años sesenta se da inicio al planteamiento de principales cambios al punto tal que en la actualidad se encuentra en su cúspide al aceptar e implementar los Estándares Internacionales de Información Financiera -NIIF-.

Tomando lo mencionado y los aspectos que se van a trabajar en el cuerpo del texto, se hace una revisión a la evolución de la práctica contable, hasta el punto de tener una fundamentación científica que toma en cuenta las necesidades de la sociedad en el país. Es por eso que el objetivo de este artículo es mostrar las contradicciones que se encuentran al traer el modelo de los estándares internacionales de países desarrollados al ámbito colombiano, recordando que estos cuentan con contextos diferentes al de nuestra sociedad, tanto a nivel económico como a nivel político, cultural y social. Para llegar a entender el conflicto que se puede generar al implementar un modelo basado en una economía neoliberal al modelo nacional, en el cual la economía se rige por el uso y explotación del suelo y sus recursos naturales.

La estructura que tiene el texto busca familiarizar al lector con el contexto contable colombiano, para lo cual está compuesto por tres partes. La primera se encuentra enfocada en generar una visión histórica de la contabilidad en Colombia para entender la evolución de la disciplina contable, tanto en el desarrollo de la práctica como el aprendizaje de los fundamentos teóricos. En segunda instancia se tratan las bases teóricas que son utilizadas en las NIIF. Como último argumento se aborda el proceso de adopción de las NIIF que se ha dado en Colombia mostrando los aspectos en los cuales difieren por el cambio de paradigma en la elaboración y presentación de la información financiera para la sociedad en diferentes contextos.

\section{Evolución histórica de la contabilidad en Colombia}

En Colombia el campo contable ha tenido un tardío despertar para contar con la instauración de la profesión contable por medio de una ley que entregara el reconocimiento de la misma por parte del gobierno y la sociedad. 
Como ya se explicó, este campo era manejado bajo el modelo español en la colonia y luego de la independencia este era desarrollado, como señala Calvo (2014), bajo el cumplimiento de las prácticas comerciales y mercantiles hasta cerca de 1940, donde se basaba en el conocimiento de las ganancias que se podían obtener en el intercambio de bienes por parte de empíricos en la materia, empíricos que no requerían ser personas con conocimiento amplio en el tema contable.

Para estos años Colombia, al igual que varios países de Latinoamérica, recibieron la visita de diferentes misiones con fines económicos provenientes principalmente de Europa, y que tenían como objetivo generar recomendaciones para estos países si se deseaban unir a las negociaciones y planes de inversión y desarrollo que se mantenían en Europa, para lo cual hacen que se cree la necesidad de tener bancos, entidades financieras y organizaciones para poder negociar con ellos, no solamente con gobiernos y ciudadanos. De esta manera es como se da en materia contable el comienzo de esfuerzos para obtener la formación y la habilitación profesional, logrando que se tengan técnicos, mecanógrafos, entre otros profesionales, siendo el logro más importante en el momento la creación de la Facultad Nacional de Contaduría.

Más adelante, en los años setenta, se da una competencia dentro del mercado laboral, el cual previamente se construyó dadas las recomendaciones de las misiones ya comentadas, esto se presenta ya que se cuenta con una formación especializada de las personas que desean desarrollar su actividad laboral en este campo, pero sobre todo se presenta la competencia debido a que las empresas e inversores extranjeros traen además de su dinero y maquinarias al personal que desarrolla el manejo de la contabilidad en las mismas, restando así oportunidades para obtener experiencia y desarrollar su potencial práctico a los egresados contables juramentados con que se cuenta en el país.

En los años ochenta, y dado el creciente número de personas que tienen formación en contabilidad, se da la necesidad de que el gobierno nacional realice control sobre la práctica contable, por ello se expide el Decreto 2160 de 1986, que para este momento reglamenta la práctica y genera los principios 
de contabilidad generalmente aceptados sobre los cuales se basarán tanto el reconocimiento de partidas como la elaboración de los estados financieros de las empresas (Congreso de la República de Colombia, Decreto 2160, Por el cual se reglamenta la contabilidad mercantil y se expiden las normas de contabilidad generalmente aceptadas, 1986). Para el inicio de la década delos años noventa y en medio de la coyuntura, debido a cambios producto de la constituyente que vivió el país, se genera la Ley 43 de 1990, la cual reglamenta la profesión del contador público además de dictar otras disposiciones sobre la entidad y la regulación que se llevará a cabo a los diferentes profesionales que la ejercen (Congreso de la República de Colombia, 1990).

Todo esto en pro de realizar una estandarización de las prácticas contables en el país, dada la reciente apertura económica de la que se gozaba con el exterior, y es así como se emite el Decreto 2649 de 1993, el cual es el reglamento general de contabilidad, ya que permite que el desarrollo de la profesión a nivel práctico se encuentre regulado y controlado al reunir las diferentes leyes y decretos que habían sido aprobados y publicados con anterioridad (Congreso de la República de Colombia, 1993), esta agrupación no incluyo la Ley 43 de 1990, dado que esta cuenta con un enfoque de regulación sobre los profesionales, más que sobre las prácticas contables.

Es de resaltar, que tal como concluye Calvo (2014), en las dos últimas décadas del periodo descrito, Colombia da el inicio de la profesión con regulación además de tener un enfoque teórico que incentiva la investigación por parte de los profesionales egresados, de igual manera Cubides enuncia que los "cambios muy importantes como la globalización y las normas internacionales empiezan a imponerse" (citado por Pinzón, 2011, p. 59), para Colombia esto se evidencia en el Decreto 2649/93, aunque la regulación en el país se encuentra basada en el Marco Conceptual de 1989 del IASC (International Accounting Standards Committee) que era aplicado en materia contable por diversos países .

Otro aspecto que ha sido de gran importancia en Colombia es el hecho que durante la evolución que la profesión ha tenido, el país se ha caracterizado por llevar una contabilidad patrimonial debido al contexto en el que se 
encuentra, donde se exige que la contabilidad proporcione información para realizar control así como garantizar el recaudo tributario, que se ha convertido en su principal fin, estando de esta manera regulado en principio por decretos fiscales y tributarios mucho más que por las leyes o decretos contables, aunque actualmente está cambiando este panorama con la aceptación que se le da a las NIIF por medio de la Ley 1314 de 2009, a la cual se hará referencia más adelante.

\section{Generalidades de los Estándares Internacionales de Información Financiera - NIIF-}

Las NIIF se basan en una economía neoliberal, dado que su objetivo a nivel económico es trabajar en beneficio de los accionistas y capitalistas de las empresas al estimular las bases de acumulación privada sin tener en cuenta las repercusiones en el ámbito político o social (Rueda, 2010). La economía neoliberal no es un tema de reciente desarrollo, su mayor expansión se observa desde finales del siglo XX, mediante la desregulación por parte del Estado en relación a los postulados del modelo, donde se supone de mejor uso la libre eficiencia del mercado para asignar y redistribuir a la sociedad.

Otra característica del neoliberalismo que se refleja en las NIIF, es el aumento de acumulación privada de la propiedad, así desregulada donde se pueda tener facilidad de comprar y vender acciones de empresas de igual forma que se haría con las propiedades de las mismas; en este punto las NIIF son el medio más fácil para que un inversionista o prestamista decida sobre realizar o no negociaciones con las entidades, puesto que generan un lenguaje financiero homogéneo a nivel internacional en busca de la globalización.

Se puede decir que el valor razonable es el mejor reflejo de la presencia del neoliberalismo en los estándares, dado que como es tratado en la NIIF 13 , se debe contar con un mercado activo, que contenga datos observables, que faciliten la fiabilidad y la transparencia de la información (Consejo de Normas Internacionales de Contabilidad, 2011). Según las características 
de la información se establecen 3 jerarquías para clasificarlas dependiendo del nivel de confianza que pueda generar la valoración, estos son:

- Nivel 1: datos observables en un mercado activo de productos idénticos.

- Nivel 2: datos observables en un mercado activo de productos similares.

- Nivel 3: datos no observables, estimaciones según algunas técnicas (mercado, ingreso, costo, etc.).

Como podemos evidenciar, el valor razonable se centra en un mercado que se caracteriza por tener gran actividad y movimiento en las transacciones, de tal manera que los precios sean capaces de establecerse según las leyes de la oferta y la demanda, considerando de este modo que el mercado dará información fiable para los accionistas o posibles inversores; partiendo de esto, Gómez y Álvarez (2013) afirman que dicho método de valoración puede garantizar la veracidad de los valores entregados en los estados financieros, sin dejar de lado las limitaciones que puedan crearse al momento de no encontrar datos observables, por lo que las empresas deben tratar al máximo de respetar la jerarquía y evitar el uso de datos de nivel 3 .

Cabe resaltar que las normas internacionales no obligan el uso del valor razonable en todos los estándares, pero aun así afecta de forma significativa el sector de minería, de intermediación, el financiero y principalmente en el agrícola, donde se debe mencionar que la NIC 41 (2011) (agricultura) establece que:

"Un activo biológico se medirá, tanto en el momento de su reconocimiento inicial como al final del periodo sobre el que se informa, a su valor razonable menos los costos de venta, excepto en el caso, descrito en el párrafo 30, donde el valor razonable no pueda ser medido con fiabilidad" (Consejo de Normas Internacionales de Contabilidad, p. 821).

Este tema tiene gran impacto en Colombia, debido a que es un país que se centra en el sector primario, por ende, la mayoría de las empresas nacionales se dedican a la extracción o explotación del suelo. 
Como podemos observar, este estándar enuncia que todas las empresas de actividades agrícolas deben llevar sus activos biológicos a valor razonable, lo que genera variaciones en mediciones futuras y que afectarán de forma directa los estados financieros según las ganancias o pérdidas, dando la posibilidad de efectuar una distribución de ganancias que aún no se han realizado, lo que puede generar un peligro en la sostenibilidad de las empresas. Aunque se menciona que cuando no sea fiable la medición se permite solamente el uso del costo en la medición inicial, pero en mediciones posteriores se exige el uso del valor razonable y por consiguiente el efecto de sus variaciones.

De igual forma Gómez y Álvarez (2013) hacen un análisis histórico del desarrollo del valor razonable en las normas (debido a las dinámicas económicas) que dio inició hace más de 50 años, empezando a aplicarse principalmente dentro de los instrumentos financieros que sí contaban con un mercado idóneo para la utilización de este método de valoración. Al pasar los años se empezó a implementar en los activos y pasivos financieros, ya en mayo de 2011 con la emisión de la NIIF 13 se aplica esta medición a otros activos además de los financieros, que no cuentan en todos los casos con las características necesarias para dicha medición.

\section{Comentarios sobre la implementación de los Estándares Internacionales de Información Financiera -NIIF- en Colombia}

El proceso de implementación inicia con la sanción de la Ley 1314 de 2009, en la cual se da por mandato realizar convergencia con estándares internacionales de aceptación mundial. En primer lugar es importante resaltar que dentro de dicha ley no se establece de forma obligatoria la aplicación de las NIIF, si no como fue mencionado, debían ser estándares internacionales y el único requisito era la aceptación mundial. Por otro lado, dentro de esta podemos observar que la información debe ser "útil para la toma de decisiones económicas por parte del Estado, los propietarios, funcionarios y empleados de las empresas, los inversionistas actuales o potenciales y otras 
partes interesadas" (Congreso de la República de Colombia, 2009, p. 1). Bajo este fragmento se contempla como prioridad un grupo amplio de usuarios que son mencionados también en el Marco Conceptual (MC) emitido por el IASC en 1989, que fue de aplicación hasta el año 2010, dada la publicación de un nuevo MC el cual establece que la información debe ser "... útil a los inversores, prestamistas y otros acreedores existentes y potenciales para tomar decisiones sobre el suministro de recursos a la entidad" (IASB, 2010, p. 26), además se dice explícitamente que la información puede ser utilizada por otros interesados, aunque esta es realizada específicamente para los usuarios mencionados. Con este cambio varía el objetivo de la implementación de los estándares internacionales en Colombia, ya que deja de lado ciertos usuarios que son importantes, entre estos el Estado.

En Colombia, aunque lo indica la ley y ya se mencionó, se debe hacer convergencia, para lo cual ha decidido hacerlo a NIIF emitidos por el IASB (International Accounting Stardards Board), y de los cuales ya se ha hablado con anterioridad. Es necesario tener claro que para realizar una convergencia como la planteada se debe llevar a cabo una negociación o poner en acuerdo diferentes cosas entre dos o más entes, que para este caso serían el IASB y el Gobierno Nacional, esto es lo que no se evidencia actualmente para nuestro país, por el contrario se observa cómo en materia contable Colombia ha decidido tomar y aplicar de manera total, general y obligatoria las normas emitidas por el IASB tanto para grandes empresas como para las Pymes, siendo así una adopción del modelo y paradigma establecido por las NIIF.

Son diversos factores los que muestran cómo el proceso de implementación de las NIIF va contra las características de un país como Colombia, puesto que el sistema contable de estos estándares internacionales se enmarca en una fuerte influencia anglosajona. Mientras el modelo contable que maneja nuestro país tiene propensión macroeconómica, es decir que las normas contables se encuentran relacionadas con las normas fiscales, el modelo internacional se inclina por lo microeconómico.

Se llega por lo tanto al concepto del sistema contable, en el cual "subyacen los conceptos, principios generales y prácticas específicas de un país 
o área geográfica determinada" (Jarne, 2001, p. 8), para así establecer con mayor franqueza cuáles son las diferencias que existen a nivel internacional en el ámbito contable. Consideramos entonces todos aquellos aspectos que intervienen en el desarrollo de este, tanto dentro del sistema (agentes internos) como en su entorno (agentes externos), ejerciendo por lo tanto cierta influencia de parte de cada uno de los factores, que al combinarse estos se crea un sistema contable único en cada país.

En su sistema económico, Colombia se presenta como un país en vía de desarrollo en comparación con otros países, en los cuales encontramos grandes empresas a las cuales es necesario aplicar estándares internacionales debido a la cantidad de información y transacciones que estas requieren para su normal continuidad; sin olvidar la importancia de la información que se produce para los inversionistas.

En Colombia, a diferencia de los países desarrollados, se cuenta con pocas empresas de gran tamaño, consideradas en el grupo 1 de la reglamentación nacional para la aplicación de los estándares, y hallamos en su gran mayoría Pymes y microempresas, sobre todo empresas familiares, que presentan financiación propia y de créditos entregados por parte de entidades financieras, evidenciando así que "la posibilidad de financiación empresarial por medio del mercado de valores sea muy poco contemplada" (Ferrer, 2013, p. 996) y teniendo en cuenta que "la participación de los agentes en el mercado de valores se ve afectada por los costos asociados a la participación del mismo" (Ferrer, 2013, p. 997) se dificulta que estas empresas intenten participar en los mercados bursátiles, en los cuales se basan los estándares internacionales, además que sin la intervención en estos mercados activos el método de valoración ya descrito que se requiere no sería una buena opción para aplicar en este tipo de empresas en cuanto a la negociación de sus propiedades.

Además la economía nacional se está pronunciando en el mercado internacional, gracias a la globalización como una "economía emergente" y está presenciando negocios e intercambios comerciales con otros países, atrayendo así inversionistas y por consiguiente los estándares internacionales, que 
se configuran como una herramienta importante para estas negociaciones. Pero aun así el camino que tiene que recorrer el país en este nuevo escenario es denso, debido a que los empresarios colombianos conocen poco o nada de las NIIF (Ministerio de Comercio, 2011).

Tomando en cuenta factores culturales, en Colombia encontramos una sociedad conservadora donde hay prudencia en la medición, confidencialidad de la información financiera, control estatal, uniformidad en la aplicación de reglas contables; mientras que las NIIF se encuentran en el contexto de modernidad de las relaciones sociales, de autocontrol, de información transparente y abierta al público en general, y de apertura de negocios tomando los riesgos que estos conllevan (Ferrer, 2013).

En aspectos como la regulación contable, es importante destacar que en Colombia el organismo emisor de normas es público: Consejo Técnico de la Contaduría Pública, distinto al organismo de carácter privado del modelo internacional en el que la regulación contable es establecida por la profesión. Y teniendo en cuenta el papel de CTCP y la JCC el proceso de adaptación que se está realizando en Colombia es diferente a la tendencia mundial, donde las funciones se dividen para ser ejecutadas por tres organismos: un organismo emisor, uno regulador y otro profesional, de naturaleza privada (Ferrer, 2013).

El objetivo de las NIIF es proporcionar información fiable para la toma de decisiones, pero como postula la autora Natalia Baracaldo (2013) "los estándares de contabilidad en sí no son garantía de veracidad contable y transparencia" (p. 1.110), pues el tema de transparencia debe ser tratado por el gobierno corporativo, por medio del control interno, de normas de auditoría y una ética profesional, pues la falta de éstas y las malas intenciones de los productores de las información financiera puede ocasionar que se cometan situaciones de fraude, que además de traer grandes consecuencias en la toma de decisiones pueden generar con ello repercusiones económicas que afecten a la empresa. 
En el modelo internacional también identificamos la separación de la contabilidad financiera con la fiscal, caso que no se presenta en Colombia, donde no se ofrece esta disociación debido a que las prácticas contables terminan acogiéndose a los criterios de las normas fiscales. En las NIIF no encontramos un plan general de contabilidad, diferente al caso colombiano donde se cuenta con un plan único de cuentas. Por otro lado, si consideramos el manejo y progreso de la profesión y la educación contable, en el ambiente nacional presenciamos varias debilidades tanto en los fundamentos como en los métodos utilizados para enseñar, sin dejar de lado la poca, casi nula, profesionalización docente en el tema contable.

Es por eso que se hacen críticas a los Estándares Internacionales de Educación (IES), debido a que para la enseñanza universitaria se presentan de forma incompleta dejando de lado ciertos aspectos como la formación en valores, no reconocen las necesidades específicas de los contextos pues pretende la homogeneización, buscan atender los mercados financieros dejando de lado la realidad productiva mundial, y además posee una perspectiva funcional e instrumental de la contabilidad (Gómez, 2007).

Es necesario entonces tener presente que se requieren de ciertos cambios en la empresa, así como en la infraestructura en comunicaciones e informática, de forma tal que se puedan llevar a cabo los procesos requeridos en la adopción del modelo de estándares internacionales para la producción de la información exigida, pues en desarrollo y cumplimiento de lo anterior es necesario contar con nueva tecnología, nuevas prácticas contables, sistemas de información y una nueva cultura organizacional. Esta última debido a que contiene diversos enfoques y características que definen si los cambios a los que se debe acoplar la empresa y sus estrategias ejecutadas pueden proceder con cierto grado de facilidad, o si por el contrario van a tender a fallar o tener inconvenientes en algunos aspectos. Dado que reconoce las capacidades intelectuales, el trabajo y el intercambio de ideas entre los individuos, de forma tal que si como resultado se llega al cambio, se puede obtener productividad en la empresa y el impulso competitivo de la misma, como se desea (Cújar, Ramos, Hernández y López, 2013). 


\section{Conclusiones}

Bajo la recopilación de ideas tratadas en el presente documento se puede señalar que las NIIF se encuentran basadas en una perspectiva neoliberal y de mercados altamente desarrollados, principalmente los financieros, las cuales al llegar al país se enfrentan a grandes conflictos por las diferencias de los paradigmas entre los modelos, en cuanto a su aplicación en contextos tan diferentes como los que presenta el mercado colombiano. Aunque se ha evidenciado un desarrollo en materia contable desde la década de los ochenta que ha permitido un mayor avance a nivel práctico y teórico, no ha sido el suficiente para que en la actualidad Colombia esté listo para hacer la aplicación del modelo NIIF, debido en parte a que la formación de la profesión ha sido más técnica, al punto que ha generado un pensamiento dentro de la sociedad de teneduría más que por el deseo de adquirir conocimiento.

Es por lo anterior que para poder aceptar y apropiarse del modelo en Colombia se necesita en principio un cambio en el uso de la información contable en diferentes aspectos de la sociedad, pero principalmente a nivel económico dado que se observa que "las razones de la íntima relación entre contabilidad y desarrollo son evidentes, si tenemos en cuenta que la primera es fuente primordial de información que, a su vez, facilita la planificación macro y micro económica" (Cubides, Maldonado, Machado, Visbal y Gracia, 1991, p. 5), y el modelo NIIF se basa en esta relación de una amplia manera, ya que se rige en buscar que los países y sus economías crezcan por medio del uso adecuado y eficiente en la sociedad de la información que las empresas publiquen, la cual debe ser transparente y confiable desde la gerencia misma.

Finalmente, se encuentra que para Colombia el gobierno ha decidido hacer obligatorio el cambio hacia las NIIF por medio de su rama legislativa con la Ley 1314/09, la cual a su vez presenta una inconsistencia entre su contenido y su finalidad, ya que está basada en el cumplimiento del MC que se encontraba vigente al momento de su expedición y no en su versión más reciente, así como indica que se deben cumplir todas las NIIF publicadas 
sin tener en cuenta que algunas no han entrado en vigencia obligatoria en aquellos países que las aplican desde tiempo atrás, lo cual podría ser contraproducente en Colombia puesto que no se tiene el suficiente conocimiento para hacer la mejor aplicación de las mismas.

Además como se ha mencionado, se tiene que el país cuenta con una economía dominada por empresas Pymes y el modelo NIIF está dirigido a aquellas entidades que cuentan con grandes capitales y tienen la capacidad de estar en mercados bursátiles, por medio de las bolsas de valores, siendo en esta aplicación el mayor impacto.

\section{Referencias}

Baracaldo, N. A. (2013). ¿Es garante del principio de transparencia la implementación de normas internacionales en Colombia? (número especial: Cuatro años de convergencia contable: resultados, transformaciones y retos). Cuadernos de contabilidad, 14(36), 1.097-1.120.

Calvo, A. P. (2014, 13 de mayo). Notas acerca de la relación Contabilidad-Sociedad. (C. d.-U. Colombia, entrevistador).

Congreso de la República de Colombia. (1986, 9 de julio). Decreto 2160, por el cual se reglamenta la contabilidad mercantil y se expiden las normas de contabilidad generalmente aceptadas. Bogotá D. C., Colombia: Diario Oficial 37.545. Congreso de la República de Colombia. (1990, 1 de diciembre). Ley 43, por la cual se adiciona la Ley 145 de 1960, reglamentaria de la profesión de contador público y se dictan otras disposiciones. Bogotá D. C., Colombia: Diario Oficial 39.602. Congreso de la República de Colombia. (1993, 29 de diciembre). Decreto 2649, por el cual se reglamenta la contabilidad en general y se expiden los principios o normas generalmente aceptados en Colombia. Bogotá D. C., Colombia: Diario Oficial 41.159.

Congreso de la República de Colombia. (2009, 13 de julio). Ley 1314, por la cual se regulan los principios y normas de contabilidad e información financiera y de aseguramiento de información aceptados en Colombia. Bogotá D. C., Colombia: Diario Oficial 47.409. 
Consejo de Normas Internacionales de Contabilidad. (2011). NIC 41, agricultura. IFRS Foundation.

Consejo de Normas Internacionales de Contabilidad. (2011). NIIF 13, medición del valor razonable. IFRS Foundation.

Cubides, H., Maldonado, A., Machado, M., Visbal, F., y Gracia, É. (1991). Historia de la contaduría pública en Colombia: siglo XX. Bogotá D. C.: Universidad Central, Departamento de Investigación.

Cújar, A. D., Ramos, C. D., Hernández, H. E., y López, J. M. (2013). Cultura Organizacional: evolución en la medición. Estudios Gerenciales, 29, 350-355. Ferrer, A. M. (2013). Análisis del proceso de convergencia a Normas Internacionales de Contabilidad e Información Financiera desde los factores intrínsecos al sistema contable en Colombia. Cuadernos de contabilidad, 14(36), 971-1.007.

Gómez, M. (2007). Las insuficiencias disciplinares de los estándares internacionales de educación -IES- para contadores profesionales. Lúmina, 8, 24-42.

Gómez, O., y Álvarez, R. (2013). Medición a valor razonable en la contabilidad financiera. Cuadernos de contabilidad, 14(35), 441-461.

IASB. (2010). Marco conceptual para la información financiera. IFRS Foundation.

IASC. (1989). El marco conceptual para la preparación y presentación de los estados financieros. IASC.

Jarne, J. I. (2001). Concepto de sistema contable y causas de las diferencias a nivel internacional. J. A. Laínez Gadea. Manual de Contabilidad Internacional, 3961. Colección Economía y Empresa/Pirámide Series.

Ministerio de Comercio. (2011). Convergencia hacia las Normas Internacionales de Información Financiera y Aseguramiento de la Información -NIIF-. Recuperado de http://www.fenalco.com.co/sites/default/files/guianiif.pdf

Pinzón, J. E. (2011). Entrevista con Humberto Cubides, uno de los autores de este libro clásico. J. E. Pinzón Pinto. Historia de la contaduría pública en Colombia siglo XX: a 20 años de su públicación, 12(30), pp. 369-380.). Cuadernos de contabilidad.

Rueda, G. (2010). Neoliberalismo y convergencia contable. Orígenes, características y propuestas. Lúmina, 11, 264-278. 